\title{
A Simple Electromagnetic Analysis of Magnetic NDE Using a Double Rectangular Coil and a Hall Effect Sensor
}

\author{
Laroussi Bettaieb, Hamid Kokabi, and Michel Poloujadoff
}

\author{
Université Pierre et Marie Curie (UPMC), Laboratoire d'Électronique et Électromagnétisme (L2E), \\ Aile 65/66 - 2ème étage - 4, Place Jussieu 75252 Paris Cedex 05. \\ laroussi.bettaieb@supelec.fr; hamid.kokabi@upmc.fr; michel.poloujadoff@upmc.fr
}

\begin{abstract}
In this paper a simple analysis and measurement in eddy current NDE are presented. A Hall probe is associated to a double Printed Circuit Board PCB. The configurations examined involve the coil in air and the coil above aluminum plates, either with or without cracks of various depths. The agreement between experimental and theoretical results is very good, showing that a very simple model accurately describes the electromagnetic fields.
\end{abstract}

Keywords: Hall Effect probe, eddy current testing, simplified analytical analysis of eddy currents, magnetic field measurement, rectangular double coil.

\section{Introduction}

The early apparatus for Electromagnetic Non Destructive Evaluation (E-NDE or E-NDT) consisted of a circular probe coil whose impedance was measured all over the surface of the metal sheets to be examined. Variations of impedance indicated the presence of flaws [1 to 6]. This method is still very popular, but many efforts are under development to increase its sensitivity. One suggestion has been to replace the impedance value by the resulting magnetic field as the basic signal.

Since the SQUID «Superconducting Quantum Interference Device» is a very attractive sensor of magnetic field intensity, it has been attempted to introduce it in the vicinity of the probe coil [7 to 13]. But, since it is subject to saturation, investigators replaced the circular coil by a double D [14 and 15]. In an excellent paper, Poulakis and Theodoulidis [16] have simplified the equipment by using a double rectangular printed circuit board coil and a SQUID. They have presented such a system, and given a series expansion analysis which is reported in [17].

In what follows, we present an experimental set up including a double rectangular coil where the SQUID is replaced by a Hall probe which is less sensitive, but may be installed very close to the area to be examined. And we give a simplified analytical model which is simpler than the above quoted one, without loss of accuracy.

\section{Analysis of the coil field}

The set up is described in figure 1. In figure 1 (A), a double rectangular coil printed circuit is shown. In figure $1(B)$, we show a conducting plate of thickness $C$, and the printed circuit above it; a Hall probe is attached as shown.

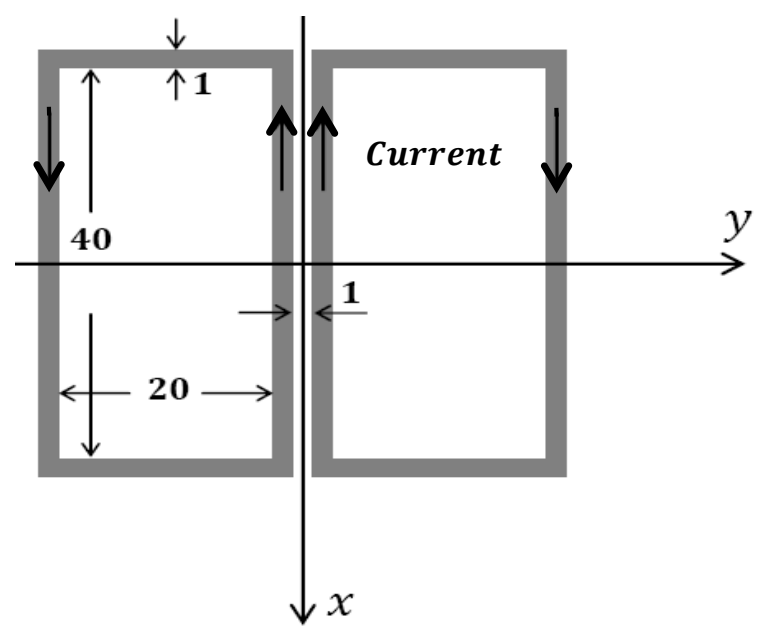

(A)

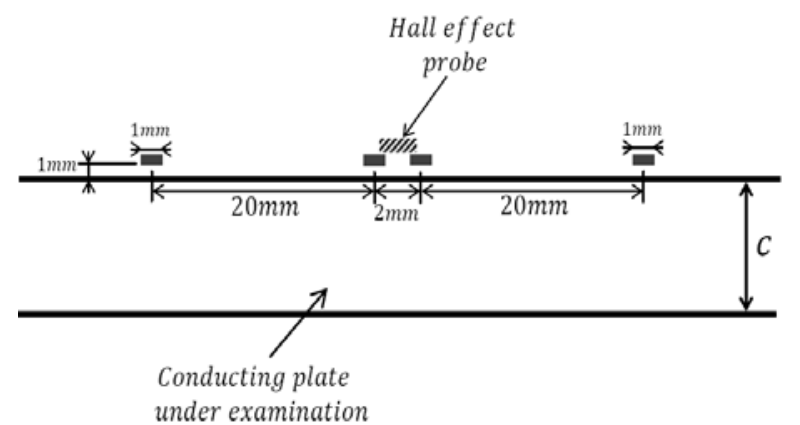

(B)

Figure 1: (A) Top view of the rectangular double printed coil; (B) Side view of this coil above an aluminum plate

We first analyze the electromagnetic field created, in free space, by a current of $1.7 \mathrm{~A}$ flowing in the rectangular double coil. This may be done by integrating the Biot and Savart formula (figure 2 (A)) 


$$
d \vec{B}=\frac{\mu_{0} I}{2 \pi} \frac{\overrightarrow{d l} \wedge \overrightarrow{P M}}{\|\overrightarrow{P M}\|^{3}}
$$

at a point $M$, where $P$ is varied along the two rectangles. As for $M$, for the purpose of this paper, it will be varied in the plane normal to figure 2 (B) and including $O y$. We considered that, if $\left(\frac{L}{l} \geq 2\right)$, it is enough to evaluate the field as if $L$ was infinite (in [18], the authors make an analogous remark, but adopt $\frac{L}{l} \geq 4$ ).

Therefore, the calculation of the coil field is reduced to a fourfold application of the Biot and Savart law for infinitely long conductors [19 to 21].

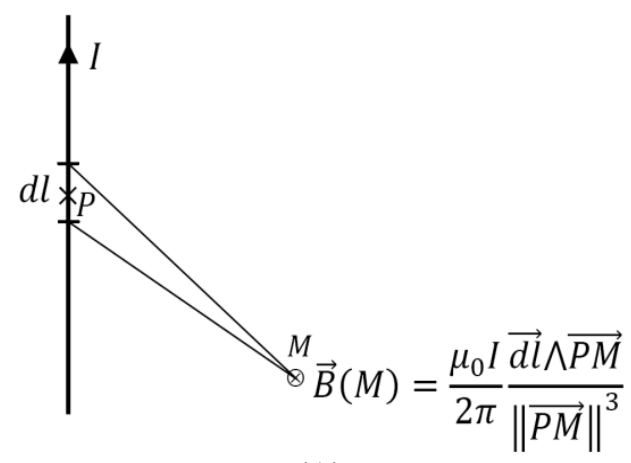

(A)

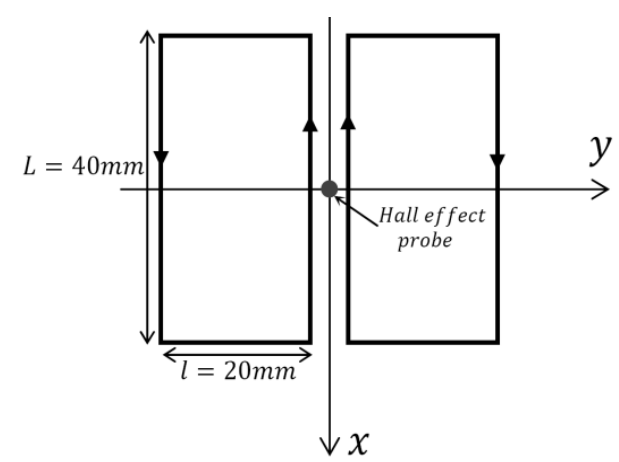

(B)

Figure 2: (A) Differential element for application of Biot and Savart law; (B) location of the Hall Effect probe

Those approximations have been carefully checked. In figure 3 , the value of the normal component of the double rectangular coil field, is described for three values $(2,8,22 \mathrm{~mm})$ of the vertical distance from the double rectangular plane (current intensity being $1.72 A$ ). There is no significant difference between experimental and theoretical values.

\section{Analysis of the currents induced in flawless plate and of the resulting $B_{z}$ field}

Assume now that the plate in figure $1 . \mathrm{b}$ has a resistivity equal to $5.82 * 10^{-8} \Omega . m$ (Aluminum $A G 3$ at $20^{\circ} \mathrm{C}$ ); its dimensions in the directions $O x, O y, O z$ are 110,100 , and $5 \mathrm{~mm}$. The current in the double rectangular coil is $1.72 \mathrm{~A}$ (as above) and its frequency is $180 \mathrm{~Hz}$. The corresponding skin depth is $9.1 \mathrm{~mm}$, much larger than the plate thickness.

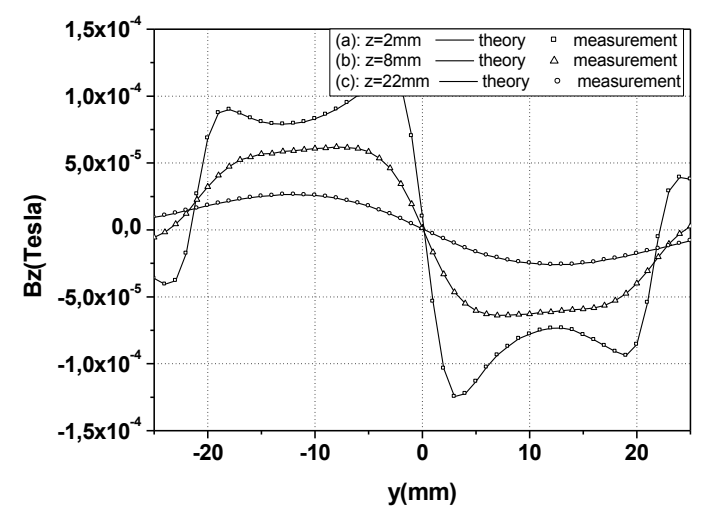

Figure 3: Magnetic field evaluation and measurement for the excitation coil in air for different values of altitude $\mathrm{z}$

Clearly, this means that the magnetic field of the currents induced in the plate is totally negligible in comparison to the exciting field [22 and 23]. Therefore, the plate can be viewed as shown in figure 4 .

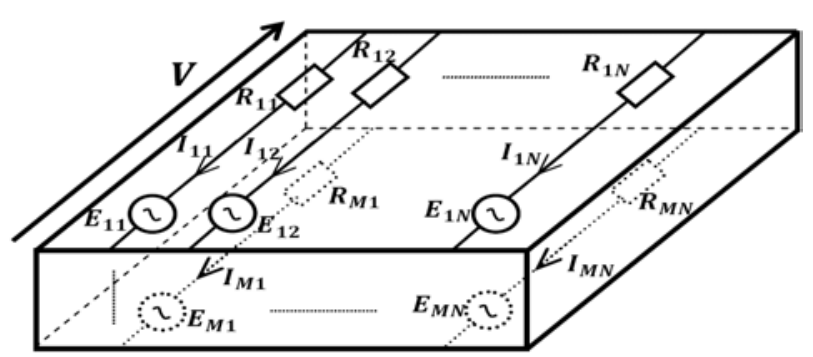

Figure 4: Model for the evaluation of emf and currents induced inside the sane conducting plate

The potential vector is parallel to Ox, and its origin may be chosen arbitrarily. If we take this origin inside the plate, just below the geometrical center of the double rectangular coil, the emf's shown in figure 4 will be clearly defined. Indeed, in figure 5, the plate is divided into $M \times N$ small elements of resistance $R_{i j}$, easy to evaluate. Let us call $V$ the potential difference between the two sides of the plate (equipotential surface). An emf $E_{i j}=-\frac{d A_{i j}}{d t}$ is created along element $(i, j)$, $A_{i j}$ being the potential vector created by the double rectangular coil along this element.

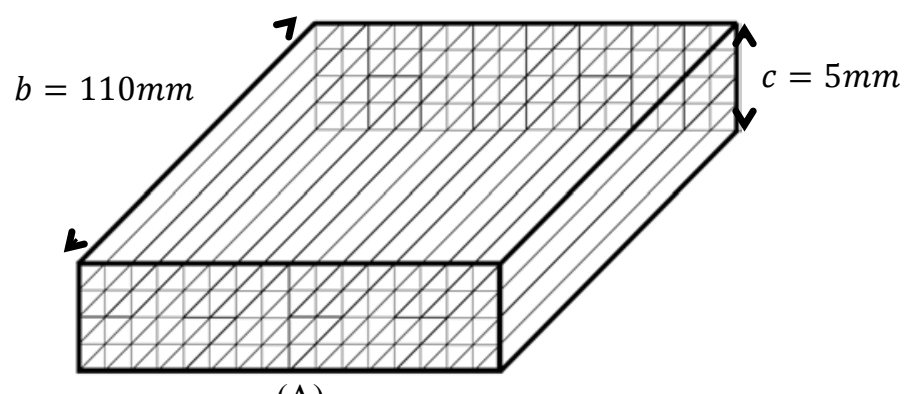

(A) 


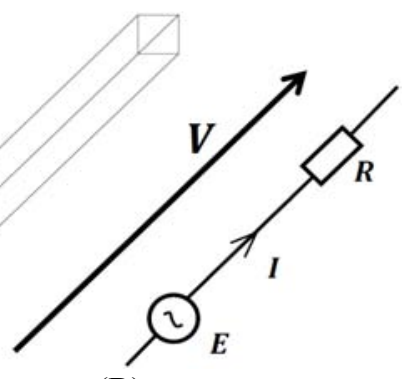

(B)

Figure 5: (A) Division of a sane plate into elements; (B) electrical model of one element

The sum of the currents in the individual conductors must be zero; therefore, if the current in element $(i, j)$ is $I_{i j}$, we have $M * N+1$ equations:

$$
\begin{aligned}
& E_{i j}-R_{i j} * I_{i j}=V \\
& \sum_{i, j} I_{i j}=0
\end{aligned}
$$

which determine $V$ and the $I_{i j}$ 's.

In figure 6 , the result of this analysis is shown for three positions of the double rectangular coil (which is $1 \mathrm{~mm}$ above the plate). Note that, in the present application, $E_{i j}$ does not significantly depends on $j$, it means that the induced currents do not depend on the variable $\mathrm{z}^{\prime}$.

The values of the induced currents corresponding to the three positions of the exciting coil are obtained with the values of $\mathrm{M}$ and $\mathrm{N}$ equal to 100 and 10 respectively. Furthermore, the theoretical calculation has reached already the convergence with these values of $\mathrm{M}$ and $\mathrm{N}$.

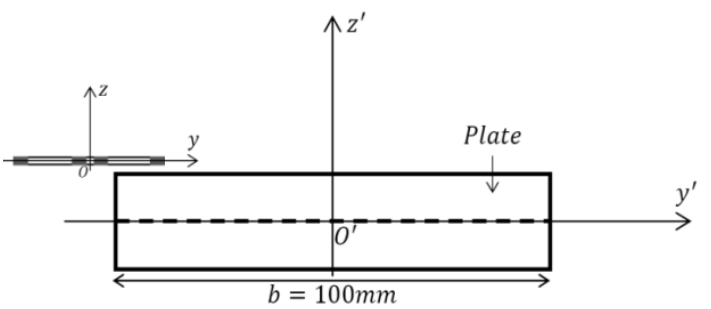

Exciting coil position 1

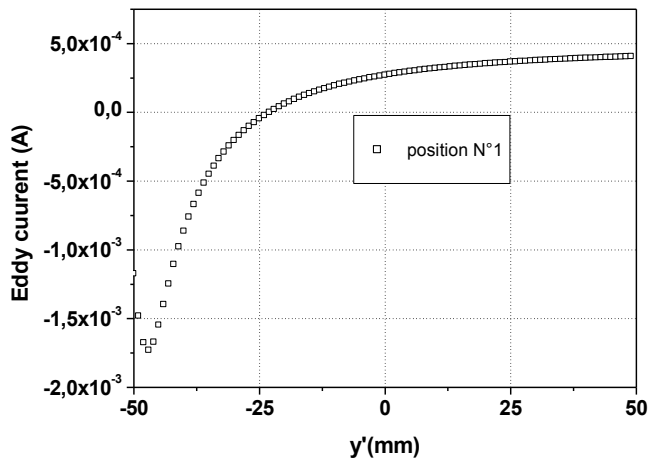

(A)

Figure 6 (A): Induced currents corresponding to the case of exciting coil position 1 as shown in the previous figure
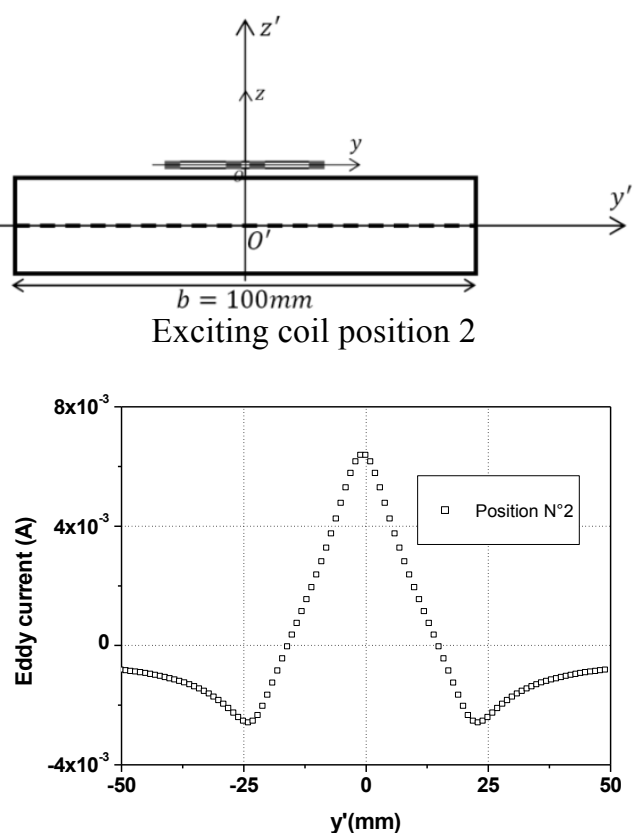

(B)

Figure 6 (B): Induced currents corresponding to the case of exciting coil position 2 as shown in the previous figure

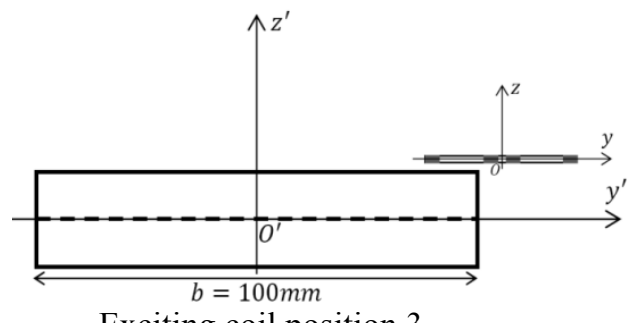

Exciting coil position 3

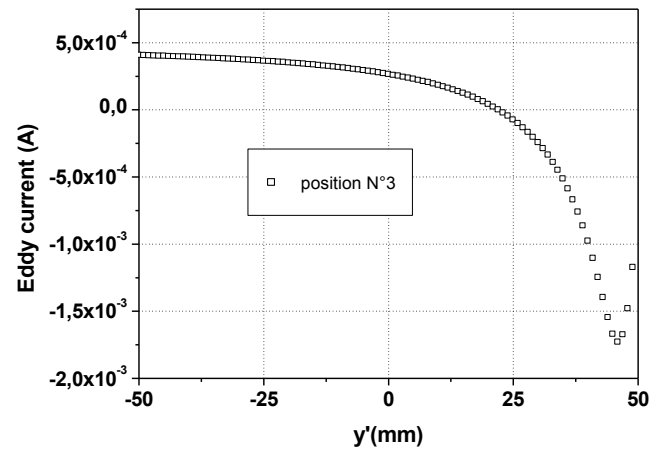

(C)

Figure 6 (C): Induced currents corresponding to the case of exciting coil position 3 as shown in the previous figure

The corresponding component of $B_{z}$ at the center of the double rectangular coil is given in figure 7 . We note that the variation curve of $B_{z}$ is clearly antisymmetric, and cancels at the center of the plate. The edges of the plate are clearly detected. 


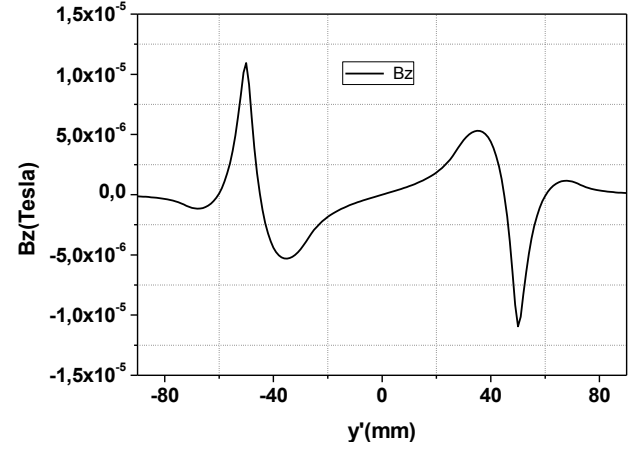

Figure 7: Computed values of $B_{z}$ for a sane plate for $y=0$ at a distance of $d=1.5 \mathrm{~mm}$ from the top surface of the plate

\section{Analysis of plate with a calibrated flaw}

Consider now two plates similar to the above one, but with a calibrated crack (figure 8). The width of the crack is $w=1 \mathrm{~mm}$, and the depths are depth $=0.5$ and $1 \mathrm{~mm}$ respectively. The induced currents are described as explained in figure 8 (A) and 8 (B).

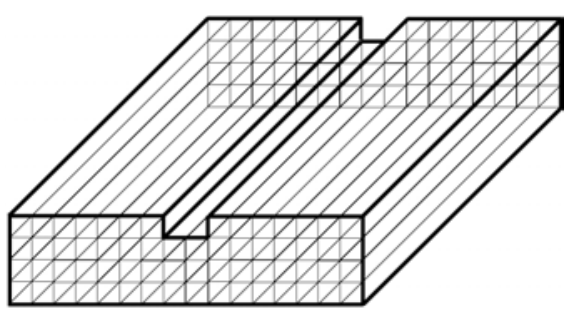

(A)

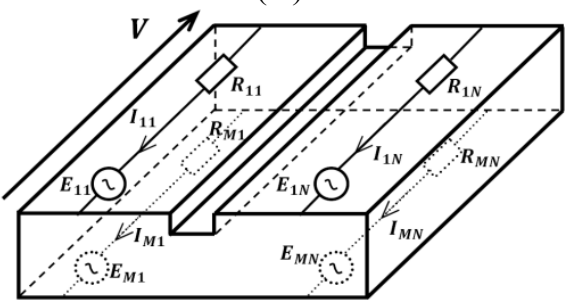

(B)

Figure 8: Same view as in figure 5, but there is a calibrated crack in the center of the plate

The distribution of the output signal $\left(B_{z}\right.$ field) at a distance of $d=1.5 \mathrm{~mm}$ from the top surface of the plate is shown in figure 9 .

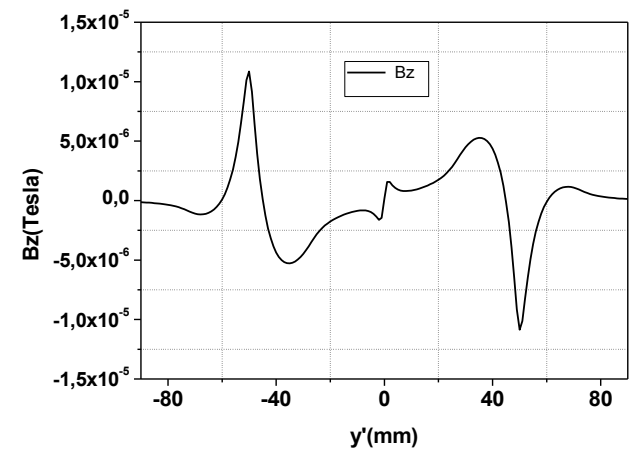

(A)

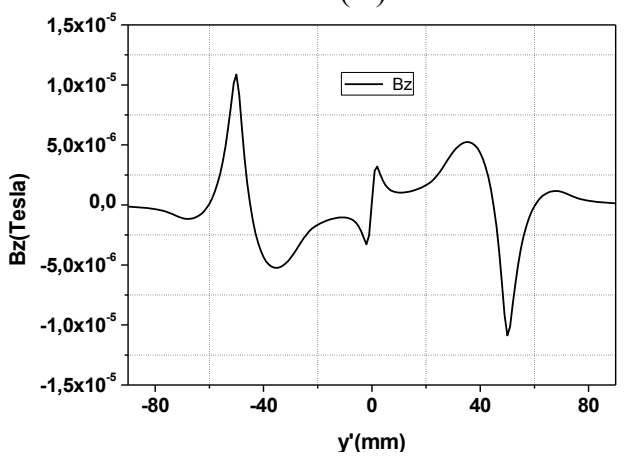

(B)

Figure 9: Same as figure 7, but with a crack width equal to $1 \mathrm{~mm}$; (A) depth $=0.5 \mathrm{~mm}$; (B) depth $=1 \mathrm{~mm}$

\section{Experimental results}

The double rectangular coil shown in figure 1.a was laid upon a glass epoxy substrate with $0.5 \mathrm{~mm}$ thickness. The copper layer thickness is $35 \mu \mathrm{m}$; its width is $1 \mathrm{~mm}$. The permissible rms value of the current is $1.72 A$ and its frequency is $180 \mathrm{~Hz}$; the total resistance is $1 \Omega$.

The Hall effect probe has been made by "ITRAN, France". Its surface is a $2.3 \mathrm{~mm}^{2}$, its volume is $0.001 \mathrm{~mm}^{3}$. The white magnetic field noise is $10 p T / \sqrt{\mathrm{Hz}}$ [24]. A synchronous detection is used, in order to distinguish the very small signal created by the eddy currents from the much larger excitation field. Its sensitivity is $5 \mathrm{mV} / \mu \mathrm{T}$ [20]. Its sensitivity is much smaller than the SQUID sensitivity, but it can operate very near the center of the double rectangular coil (the active part of a SQUID must be distant of more than $1 \mathrm{~cm}$ of the same point) [22].

At the center of the sane plate $\left(-25 \mathrm{~mm} \leq y^{\prime} \leq+25 \mathrm{~mm}\right)$, the theoretical and experimental values of $B_{z}$ are given in figure 10 at a distance of $d=1.5 \mathrm{~mm}$ from the top surface of the plate. Figure 10 shows that the measurement results tally very well with the theoretical one.

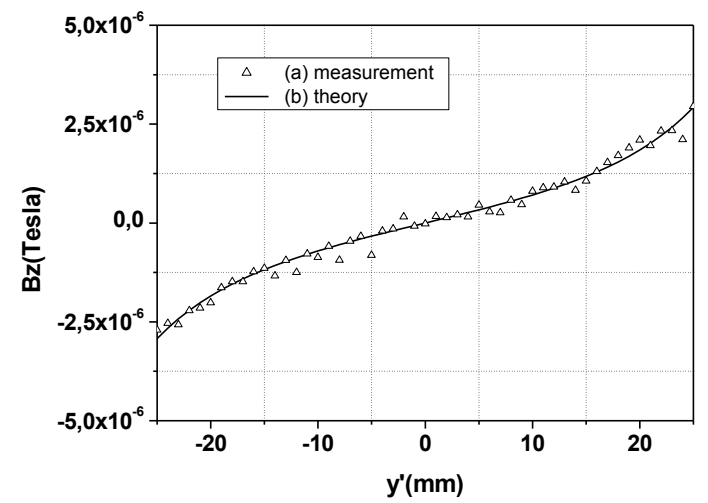

Figure 10: Comparison of the measurement (a) and the theoretical (b) values of $B_{z}$ in the center of a sane plate

When there is a calibrated crack at the center of the plate, the predicted component field $B_{z}$ is described in figure 11 (A) for a width $w=1 \mathrm{~mm}$ and for four different depths 
(depth $=0.5,1,2$ and $3 \mathrm{~mm})$. The corresponding measured values are described in figure 11 (B) at a distance of $d=1.5 \mathrm{~mm}$ from the top surface of the plate.

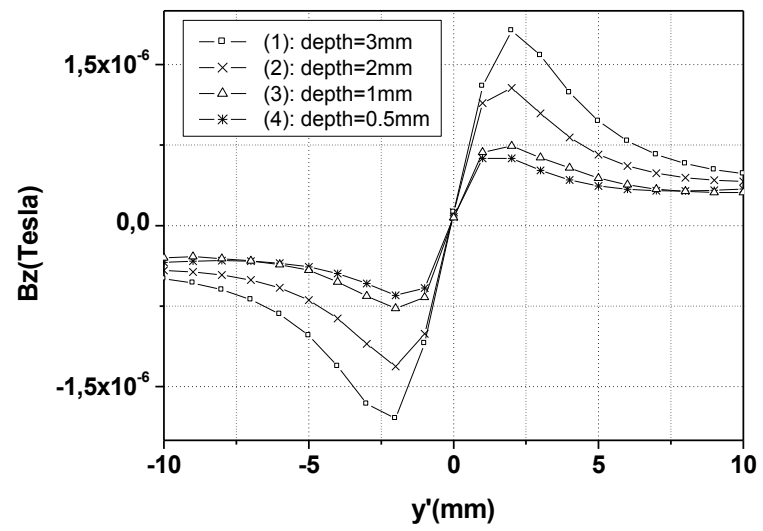

(A)

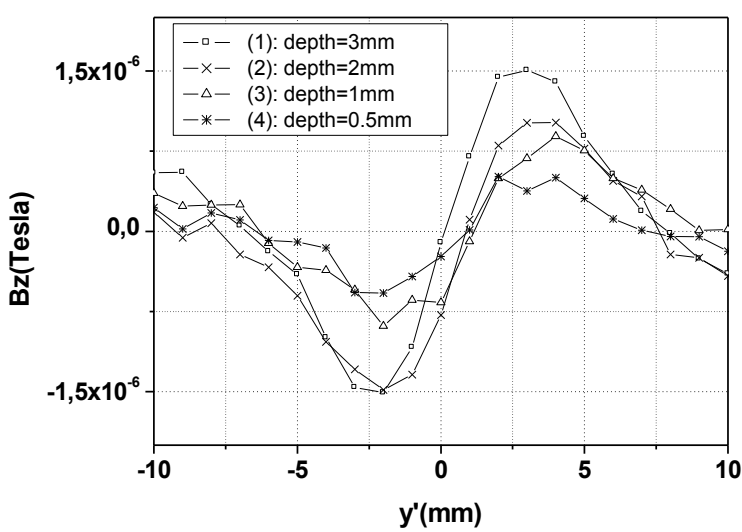

(B)

Figure 11: Comparison of theoretical (A) and measurement (B) values of the component field $B_{z}$ in the case of a calibrated crack in the center, for four different values of the crack depth ((1): $3 \mathrm{~mm},(2): 2 \mathrm{~mm},(3): 1 \mathrm{~mm}$ and (4): $0.5 \mathrm{~mm})$

If we compare the measurement results obtained in the four cases, we see that the shapes of the component field $B_{z}$ curves are totally identical and tally very well with the corresponding theoretical results.

The use of a double rectangular coil with a Hall Effect probe for detecting flaws on the surface of metallic sheets has been validated. In fact, the theoretical calculation and the measurement results are very close. Also, the RF-SQUID has been tested in previous works with the double rectangular coil [25 and 26] and consistent results have been found. In the configuration described in this paper, the use of the Hall probe seems more advantageous than the use of the SQUID. Indeed, the Hall Effect sensor, operating at room temperature, can be positioned very near the center of the double rectangular coil but the active part of our SQUID system is distant of more than $1 \mathrm{~cm}$ of the same point because of the thickness of the cooling Dewar.

\section{Conclusion}

In this paper, we describe the development of a simple, but accurate, theoretical treatment of eddy current analysis of planar samples with and without flaws. We have carefully tested the computation technique by eddy current scanning experiments using a Hall probe. The agreement between experimental and theoretical results is very good, showing that our novel model-based calculations describe well the signals obtained when using a double rectangular excitation coil and a Hall Effect probe.

\section{References}

C. V. Dodd, W. E. Deeds, Analytical solutions to eddy-current probe-coil problems, Journal of Applied Physics, vol. 39, $\mathrm{n}^{\circ}$ 6, pp. 2829-2838, 1968.

[2] C. V. Dodd, W. E. Deeds, Integral solutions to some eddy current problems, International Journal of Nondestructive Testing, vol. 1, pp. 29-90, 1969.

[3] S. M. Nair, J. H. Rose, Electromagnetic induction (eddy currents) in a conducting half-space in the absence of in homogeneities: a new formalism, Journal of Applied Physics, vol. 68, $\mathrm{n}^{\circ}$ 12, pp. 5995-6009, 1990.

[4] J. O. Fava, and M. C. Ruch, Calculation and simulation of impedance diagrams of planar rectangular spiral coils for eddy current testing, NDT\&E International, vol. 39, pp. 414-424, 2006.

[5] T. P. Theodoulidis, and E. E. Kriezis, Impedance evaluation of rectangular coils for eddy current testing of planar media, NDT\&E International, vol. 35, pp. 407-414, 2002.

[6] J. R. Bowler, and T. P. Theodoulidis, Coil impedance variation due to induced current at the edge of a conductive plate, Journal of physics D: Applied Physics, vol. 39, pp. 2862-2868, 2006.

[7] H. Weinstock and M. Nisenoff, Non-destructive evaluation of metallic structures using a SQUID gradiometer, SQUID '85, Proc. 3rd International Conference on Superconducting Quantum Devices, Hahlbohm H.D. and Lubbig H., Eds., 843--847, de Gruyter, Berlin, 1985.

[8] H. Weinstock, SQUID Sensors: fundamentals, fabrications and applications, NATO ASI series E: Applied Science, Kluwer Academic Publishers, 1996.

[9] J. Clarke, SQUIDS, Scientific American, pp. 46-53, 1994. 
[10] G. B. Donaldson, SQUID sensors, Kluwer, Dordrecht, NL, pp. 599-628, 1996.

[11] J. P. Wikswo, SQUID magnetometers for biomagnetism and nondestructive testing: important questions and initial answers, IEEE Trans. Appl. Supercond, vol. 5, n², pp. 74-120, 1995.

[12] J. Clarke, A. I. Braginski, The SQUID Handbook: Fundamentals and Technology of SQUIDs and SQUID Systems, Volume I, Wiley-VCH Verlag GmbH\&Co, KGaA, Weinheim, 2004 et 2006.

[13] W. G. Jenks, S. S. H. Sadeghi and J. P. Wiskswo, SQUIDS for NDE, J. Phys. D 30, pp. 293-323, 1997.

[14] J. A. Lobera-Serrano, J. R. Claycomb, J. H. Miller, K. Salama, Hybrid double D sheet-inducer for SQUID based NDT, IEEE Trans. On Mag., vol. 11, no. 1, pp. 1283-1286, 2001.

[15] D. Hong-Sheng, J. Zhong-Sheng, L. Li-Jun, Development of high Tc SQUID system for nondestructive evaluation, Journal of superconductivity and novel magnetism, vol. 21, no. 4, pp. 253-257, 2008.

[16] Poulakis, Theodoulidis,www.ndt.net/article/hsndt20 07/files/Poulakis_Theodoulidis.pdf

[17] J. R. Bowler and T. Theodoulidis, Coil impedance variation due to induced current at the edge of a conductive plate, Journal of Physics D: Applied Physics, 39:2862--2868, 2006.

[16] M. Ya. Antimirov, A. A. Kolyshkin, and R. Vaillancourt, Application of a perturbation to the solution of eddy current testing problems, IEEE Trans. on Mag., vol. 30, no. 3, pp. 1247-1252, 1994.

[19] N. Tsopelas, N. J. Siakavellas, Performance of circular and square coils in electromagnetic-thermal non-destructive inspection, NDT\&E International vol. 40, pp. 12-28, 2007.

[20] A. I. Rusinov, High precision computation of solenoid magnetic fields by Garrett's methods, IEEE, Trans. on Mag., vol.30, n 4, 2004.

[21] J. T. Conway, Exact solutions for the magnetic fields of axisymmetric solenoids and current distributions, IEEE Trans. on Mag., vol. 37, $\mathrm{n}^{\circ} 4$, 2001, Research in nondestructive evaluation, vol. 20, no. 3, pp. 159-177, 2009.

[22] L. Bettaieb, H. Kokabi, M. Poloujadoff, A. Sentz, H. J. Krause, and C. Coillot, Comparison of the use of SQUID an Hall effect sensors in NDE, Materials Evaluation, vol. 68, n5, pp. 535-541, 2010.

[23] L. Bettaieb, H. Kokabi, M. Poloujadoff, A. Sentz, and H. J. Krause, Analysis of some non destructive evaluation experiments using eddy current, Research in Nondestructive Evaluation, vol. 20, no. 3, pp. 159-177, 2009.

[24] P. Leroy, C. Coillot, V. Mosser, A. Roux and G. Chanteur, Use of magnetic concentrator to highly improve the sensitivity of Hall effect sensors, Sensor_Letters, vol. 5, $\mathrm{n}^{\circ}$ 1, pp. 162-166, 2007.

[25] F. Sarreshtedari, S. Razmkhah, N. Hosseini, K. Mehrany, H. Kokabi, H. J. Krause, J. Schubert, M. Banzet, and M. Fardmanesh, Analytical model for the extraction of flaw-induced current interactions for SQUID NDE, IEEE Transactions on Applied Superconductivity, vol. 21, no. 4, pp. 3442-3446, 2011.

[26] Mehdi Fardmanesh, Farrokh Sarreshtedari, Arash Pourhashemi, Elnaz Ansari, Mohammad A. Vesaghi, Juergen Schubert, Marko Banzet, and H. J. Krause, Optimization of NDE Characterization Parameters for a RF-SQUID Based System Using FEM Analysis, IEEE Transactions on applied Superconductivity, vol. 19, no. 3, pp. 791-795, 2009. 